\title{
Case Studies of Aircraft Contrails Using the 1.375-micron Channel and IR Emission Channels on MODIS
}

\author{
Bo-Cai Gao \\ Remote Sensing Division, Code 7212, Naval Research Laboratory, 4555 Overlook Avenue, SW, Washington, DC 20375 USA \\ Telephone: (202)767-8252 \\ Fax: (202)404-8894 \\ E-mail: gao@rsd.nrl.navy.mil \\ William R. Ridgway, Rong Rong Li \\ Science Systems and Applications, Inc., Code 913, NASA Goddard Space Flight Center, Greenbelt, MD 20771 USA \\ Ping Yang \\ Goddard Earth Science and Technology Center, Code 913, NASA/GSFC, Greenbelt, MD 20771 USA
}

\section{INTRODUCTION}

The emissions from subsonic jet aircraft flying near the upper tropopause can lead to the formation of condensation trails (contrails). When the relative humidity near the upper troposphere is high, contrails can persist for a long time and embed in ordinary cirrus clouds. In recent years there has been interests in assessing the radiative effects of contrails on the Earth's radiation budget. Imaging data collected with NOAA AVHRR and GOES types of satellite instruments infrequently show aircraft contrails [1]. The 36-channel MODIS instrument [2] [3] on the Terra Spacecraft has improved radiometric sensitivities and spatial resolution. It makes the task of contrail detection much easier, particularly with the 1.375-micron cirrus-detecting channel [4]. In this extended abstract, we present examples of contrail observations from MODIS data.

\section{BACKGROUND}

Contrails are sometimes detectable in AVHRR bright temperature (BT) difference images between 11 micron and 12 micron channels. They are frequently undetectable in GOES BT difference images. Previously, we made preliminary studies on contrail detection using the high spatial resolution images $(50 \mathrm{~m})$ acquired with the MODIS Airborne Simulator (MAS) instrument from an NASA ER-2 aircraft at an altitude of $20 \mathrm{~km}$. We also degraded high spatial resolution images to coarser resolution images. It was found that many contrails seen in the 50-m resolution images were also seen in the $250-\mathrm{m}$ resolution images, but not in images having a 1-km AVHRR resolution and a 4-km GOES-8 resolution. The ability to detect contrails really depends on the spatial resolution of imaging data and radiometric sensitivities of instruments. The AVHRR and GOES types of instruments are not ideally suited for routine observations of aircraft contrails because of limited instrument sensitivities and coarse spatial resolution.
The MODIS instrument on the Terra Spacecraft has 36 channels covering a spectral region between 0.4 and 14 micron. The images of the 1.375-micron cirrus detecting channel, which usually do not have surface and low level cloud contaminations, is useful for contrail detection during the daytime. The MODIS IR emission channels have 12 bits of digitization, while AVHRR channels have only $8-10$ bits. The increased MODIS radiometric sensitivities will also allow better detection of contrails from IR images.

\section{SAMPLE RESULTS FROM MODIS DATA}

We have made a number of case studies on contrail observations from MODIS data. The results from one case study of MODIS data acquired over the middle-west part of the United States on August 22, 2000 at UTC 1800 are presented below.

Figure 1 shows a portion of the 0.645 -micron channel image acquired over the States of Oklahoma and Texas. There were many commercial airplanes flying through this area. Small white cumulus clouds and large fuzzy-looking cirrus clouds are seen in this image. Linear or quasi-linear aircraft contrails are not clearly seen in this image. Figure 2 shows a 0.865 micron image over the same area. The small cumulus clouds are still observable. The thin cirrus clouds are less obvious because of increased land surface reflectances at 0.865 micron.

Figure 3 shows the 1.375-micron MODIS image over the same area. The surface and low-level cumulus clouds are not seen because of strong water vapor absorption at 1.375 micron. The upper level thin cirrus clouds and linear or quasilinear aircraft contrails are obviously seen. This image clearly demonstrates that the 1.375 -micron channel is very useful for detecting thin cirrus clouds and aircraft contrails during the daytime. By examining the shapes of the cirrus clouds in this image, one can speculate that all the cirrus clouds might have originated from narrow aircraft contrails, and subsequently aged and broadened out. If airplanes did not fly through this area, the cirrus clouds might not have been formed at all. 


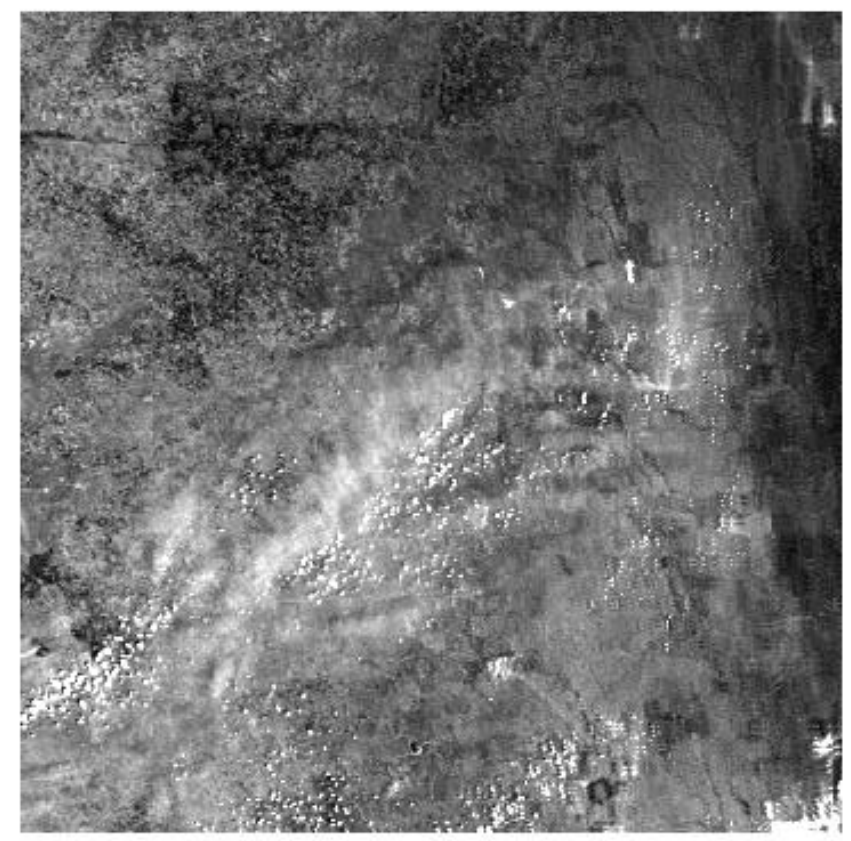

Fig. 1: A portion of a MODIS 0.645-micron channel image acquired over the States of Oklahoma and Texas. The image was taken on August 22, 2000.

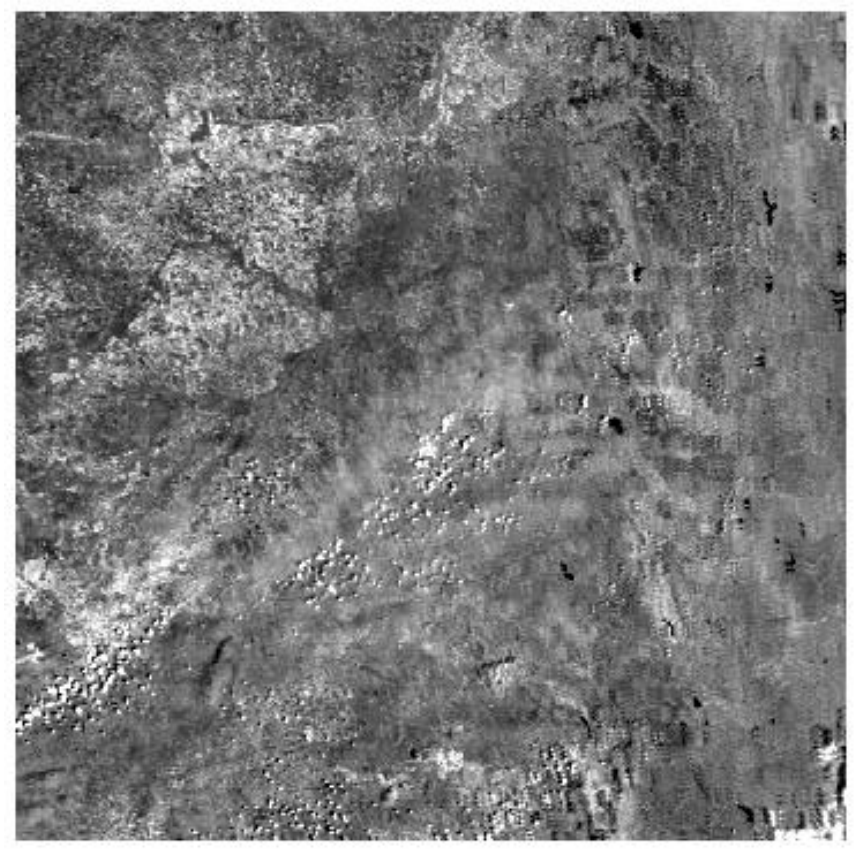

Fig. 2: A portion of a MODIS 0.865-micron channel image acquired over the States of Oklahoma and Texas.

Figure 4 shows the brightness temperature image of the 3.75-micron MODIS channel over the same area. Cirrus clouds are in the darker area of this image. Contrails are not seen in this image.

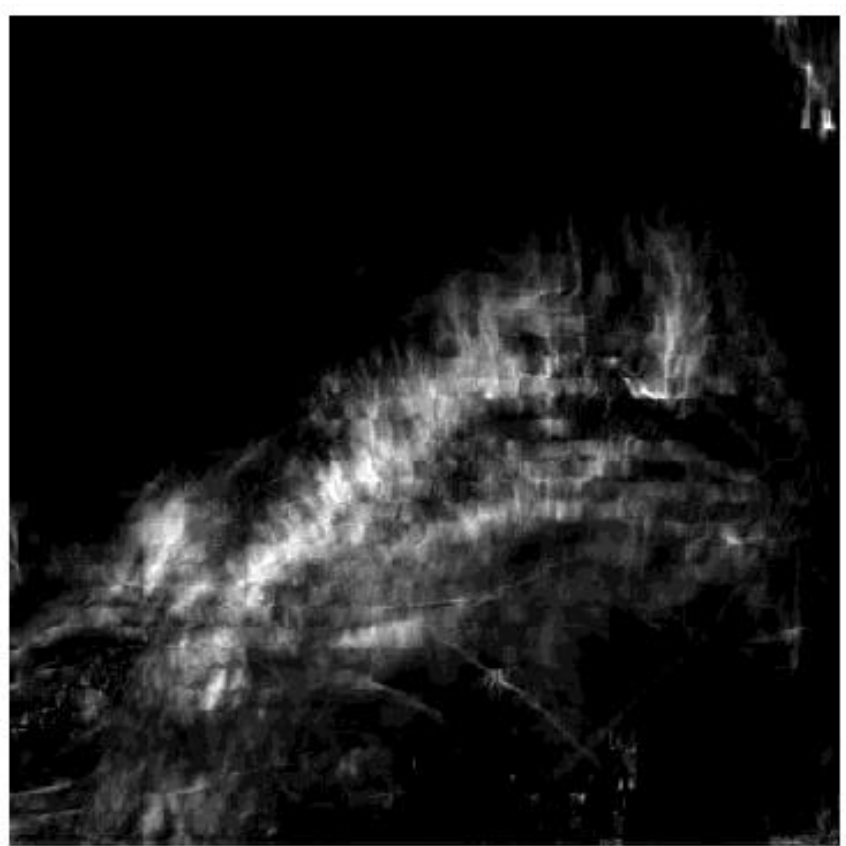

Fig. 3: A portion of a MODIS 1.375-micron channel image acquired over the States of Oklahoma and Texas.

Figure 5 shows the bright temperature image of the 11micron MODIS channel. Cirrus clouds and contrails are both seen in the darker parts of the image. The improved radiometric sensitivity of the MODIS channel (in comparison with a similar AVHRR channel) allows the detection of cirrus clouds and contrails. In order to make qualitative comparison with 1.375-micron image in Fig. 3, we show in Figure 6 the reverse of the 11-micron image. Cirrus clouds and contrails are seen clearly in this image. Some of the lower level cumulus clouds and surface features are also seen. Therefore, the 1.375-micron channel does a better job in cirrus and contrail detection than the 11-micron channel.

\section{DISCUSSIONS}

During our investigations on detecting aircraft contrails, we visually examined well over one thousand 1.375-micron MODIS images degraded to a spatial resolution of $4 \mathrm{~km}$. We have concluded that it is very difficult to positively identify aircraft contrails from the $4 \mathrm{~km}$ spatial resolution images. In some cases, we observed linear features (or "ridges") in the 4$\mathrm{km}$ spatial resolution images. After further examining the corresponding 1-km spatial resolution MODIS images, however, we realized that the "ridges" did not appear to be aircraft contrails. They were most likely to be regular cirrus clouds. Therefore, we feel that the 4-km GOES images are not well suited for positive identifications of aircraft contrails.

Previously, we developed an automated contrail detection algorithm based on the work of Englestad et al. [1] using the 
Hough transform technique. The algorithm worked quite well for the 50-m resolution MAS data. However, the same algorithm does not work well for the $1-\mathrm{km}$ spatial resolution MODIS image. Further refinement to our algorithm is needed.

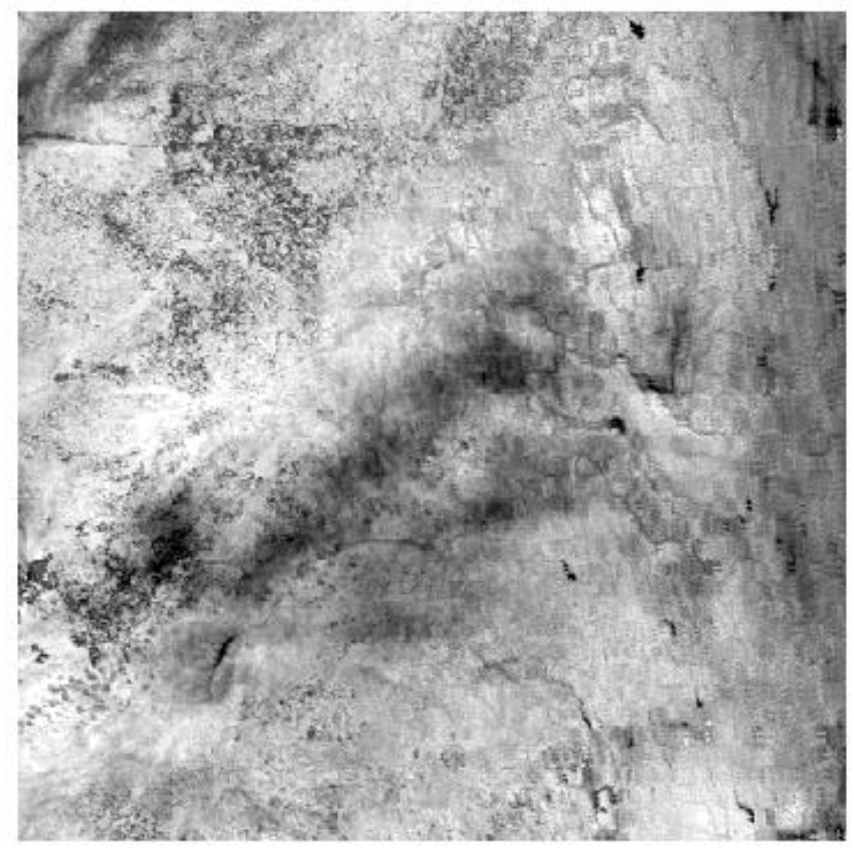

Fig. 4: A portion of a MODIS 3.75-micron channel bright temperature image.

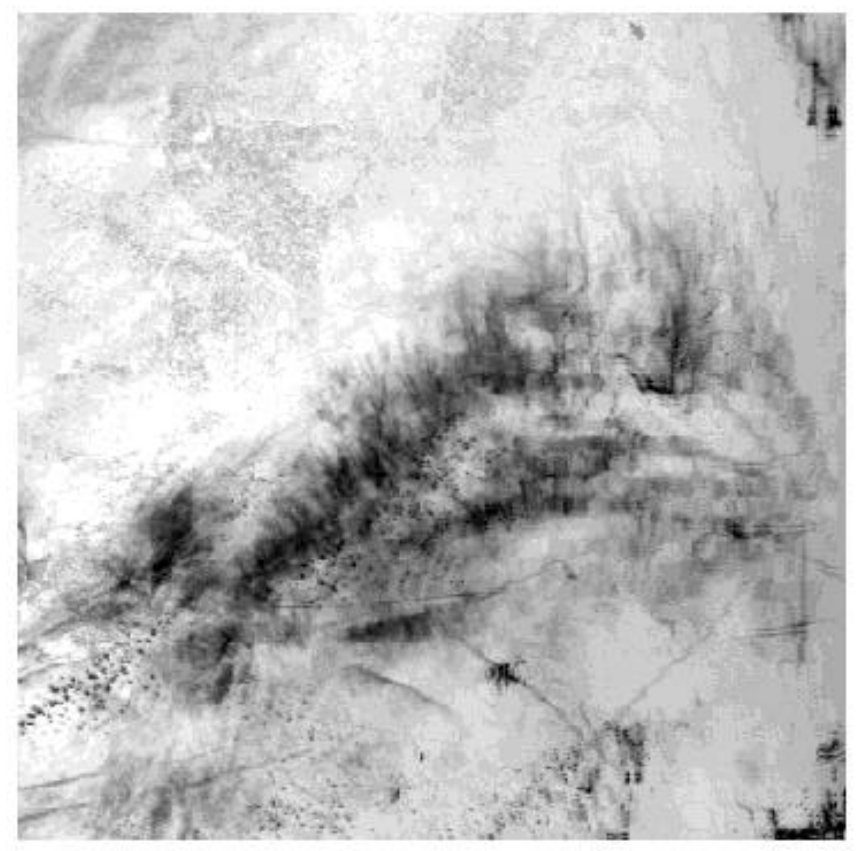

Fig. 5: A portion of a MODIS 11-micron channel bright temperature image.

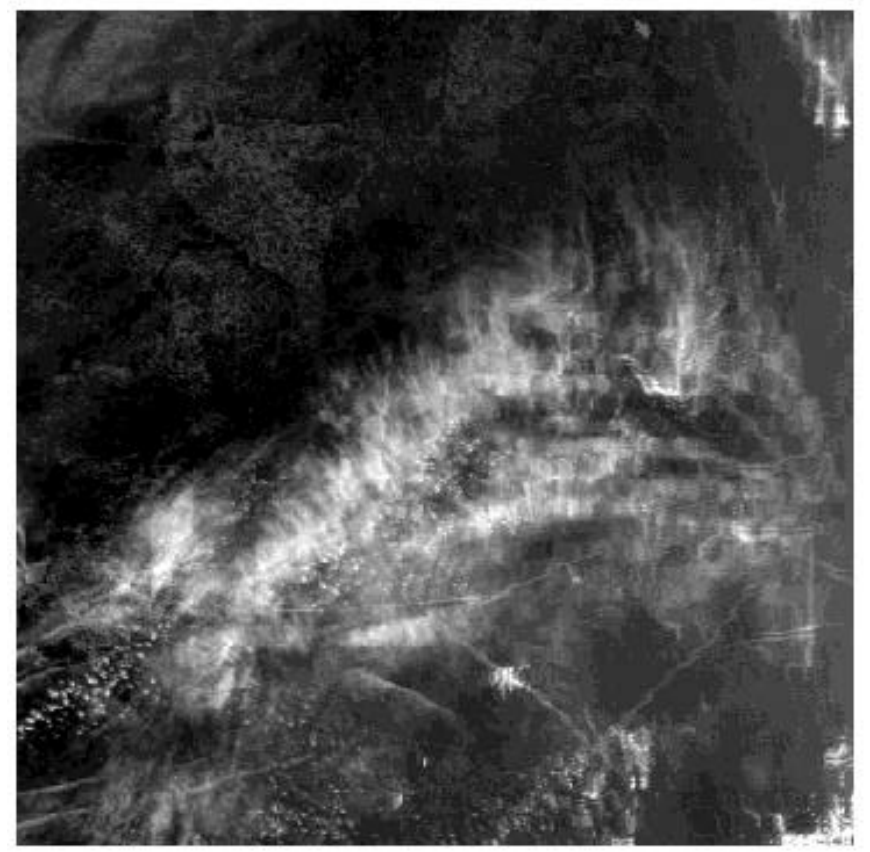

Fig. 6: The reverse of the Fig. 6 bright temperature image.

\section{SUMMARY}

Through analysis of MODIS data, we have found that the 1.375-micron channel is very useful for detecting cirrus clouds and aircraft contrails. Such detection can also be made using IR emission channels in the $10-12$ micron spectral region.

\section{ACKNOWLEGEMENTS}

This research was supported by the NASA Goddard Space Flight Center and by the Office of Naval Research.

\section{REFERENCES}

[1] Engelstad, E., S. K. Sengupta, T. Lee, and R. M. Welch, Automated detection of jet contrails using the AVHRR split window, Int. J. Rem. Sens., vol. 13, pp. 1391 - 1412, 1992.

[2] Salomonson, V. V., W. L. Barnes, P. W. Maymon, H.E. Montgomery, and H. Ostrow, "MODIS: Advanced facility instrument for studies of the earth as a system," IEEE Trans. Geosci. Remote Sens., vol. 27, pp. 145-153, 1989.

[3] King, M. D., Y. J. Kaufman, W. P. Menzel, and D. Tanre, "Remote sensing of cloud, aerosol and water vapor properties from the Moderate Resolution Imaging Spectrometer (MODIS)," IEEE Trans. Geosci. Remote Sens., vol. 30, pp. 2-27, 1992.

[4] Gao, B.-C., and Y. J. Kaufman, Selection of the $1.375-\mu \mathrm{m}$ MODIS channel for remote sensing of cirrus clouds and stratospheric aerosols from space, J. Atm. Sci., 52, 42314237, 1995. 